\title{
Nuclear Data for Reactor Physics: Cross sections and level densities in the actinide region
}

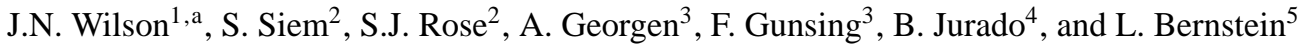 \\ 1 Institut de Physique Nucleaire Orsay, bat. 100, 15 rue G. Clemenceau, 91406 Orsay cedex, France \\ 2 University in Oslo, Blindern, Sem Sælandsvei 24, 0317 Oslo, Norway \\ 3 IRFU, Orme des Merisiers, bat. 703, 91191 Gif-Sur-Yvette cedex, France \\ 4 CENBG Bordeaux, Chemin du Solarium - Le haut vigneau. BP 120, 33175 Gradignan Cedex, France \\ 5 Lawrence Livermore National Laboratory 7000 East Avenue, Livermore, CA 94550, USA
}

\begin{abstract}
Nuclear data in the actinide region are particularly important because they are basis behind all simulations of nuclear reactor core behaviour over both long time scales (fuel depletion and waste production) and short time scales (accident scenarios). Nuclear reaction cross sections must be known as precisely as possible so that core reaction rates can be accurately calculated. Although cross section measurements in this region have been widely performed, for certain nuclei, particularly those with short half lives, direct measurements are either very difficult or impossible and thus reactor simulations must rely on theoretical calculations or extrapolations from neighbouring nuclei. The greatest uncertainty in theoretical cross section calculations comes from the lack of knowledge of level densities, for which predicted values can often be incorrect by a factor of two or more. Therefore there is a strong case for a systematic experimental study of level densities in the actinide region for the purpose of a) providing a stringent test of theoretical cross section calculations for nuclei where experimental cross section data are available and $b$ ) for providing better estimations of cross sections for nuclei in which no cross section data are available.
\end{abstract}

\section{Introduction}

There are currently around 400 nuclear reactors operating around the world producing some $16 \%$ of the world's electricity. It is likely that nuclear power will undergo a large expansion in the decades to come due to growing energy demand of a growing world population, concerns about climate change and $\mathrm{CO}_{2}$ emissions, economic growth, the depletion of reserves of oil and gas, and also for reasons of energy independence. Moreover, there is currently no other available technology that emits little $\mathrm{CO}_{2}$ and can be scaled up quickly and efficiently enough to attack the aforementioned problems in a significant way.

This expansion is now already beginning, with the construction of the latest generation of nuclear power plants (generation III) under way in China, Finland, France, India and Japan. Countries such as the USA, UK and Switzerland have construction of new reactors planned, and countries such as Sweden, Italy and Australia are seriously considering such a possibility.

However, constraints on cheaply available uranium resources, and concerns about the large quantities of nuclear waste produced by current pressurized (PWR) reactors which are often run in open-cycle mode are driving the technology to an eventual transition to more advanced reactor designs (generation IV[1]). Generation IV is an international

\footnotetext{
a e-mail: wilson@in2p3.fr
}

collaboration organized to carry out the research and development needed to establish the feasibility and performance capabilities of the next generation nuclear energy systems. It was established in 2001 and has thirteen member countries.

These reactors require many different technological innovations and hold out the prospect of nuclear power which is safer, more efficient in using uranium resources, more economical and with a greater proliferation resistance. In theory, breeder reactors (some of the Generation IV concepts) will produce more fissile material than they consume and hence could reduce the amount of natural uranium required for their operation by a factor of 60 ore more.

The immense cost of prototype reactors ( $>10^{10}$ euros) makes casual, every-day experimentation prohibitively expensive. However, thanks to the advances of computer technology over the past 30 years it is now possible to fully simulate in detail the cores of both existing nuclear reactor cores and new innovative designs. These simulations are only as good as the nuclear data on which they rely. The simulations require cross section information over huge ranges in energy $\left(10^{-3} \mathrm{eV}\right.$ to $\left.10^{7} \mathrm{eV}\right)$, and over a large number of isotopes $(>500)$. Although very accurate $(<2 \%)$ measurements of reaction cross sections for certain key nuclei have been performed (e.g. ${ }^{235} \mathrm{U},{ }^{238} \mathrm{U},{ }^{239} \mathrm{Pu}$ ) there are many nuclei where data is either inaccurate $(>20 \%)$, partial (over only a small part of the energy range needed),

This is an Open Access article distributed under the terms of the Creative Commons Attribution-Noncommercial License, which permits unrestricted use, distribution, and reproduction in any noncommercial medium, provided the original work is properly cited. 
or in some cases, particularly for short-lived nuclei, completely non-existent.

Nonetheless, reactor physics simulations require a value for the cross sections over large energy ranges for large numbers of nuclei, and any value for the cross section at a given energy, even a highly inaccurate value, is better than no value at all. For this reason the evaluated data bases (e.g. ENDF, JEFF, JENDL) have been constructed whereby measured nuclear data, extrapolations and theoretical calculations are mixed together to provide simulations with the best available information.

\section{Reactor simulations}

\subsection{Nucleosynthesis in a reactor core}

Neutron fluxes in a reactor core are relatively low $\left(10^{13} \mathrm{~cm}^{2} \mathrm{~s}^{-1}\right)$ compared to those in the most violent astrophysical processes such as supernaovae $\left(10^{28} \mathrm{~cm}^{2} \mathrm{~s}^{-1}\right)$ and as such most of the nucleosynthesis occurring in nuclear reactors occurs near the line of stability. The nuclear fissile and fertile isotopes in the fuels are in the actinide region and nucleosynthesis proceeds during the irradiation time of the fuel (typically 3-5 years) through a complex network of reactions and decays to produce new elements and isotopes. The principal nuclear reactions involved are $(n, \gamma),(n, 2 n)$ and ( $n$, fission), the latter causing the production of fission products, a multitude of mostly short-lived isotopes to the right of the stability line. In addition there are the reactors structural materials which can undergo nuclear reactions, such as $\mathrm{Fe}, \mathrm{Ni}, \mathrm{Zr}$ etc. and light elements, such as Hydrogen, Oxygen and Carbon, which can be present in, for example, the coolant and/or moderator.

\subsection{Reactor criticality, other performance parameters and safety margins}

The uncertainty in the nuclear data have a direct impact on the safety margins of the reactor principally through the uncertainty in the criticality. The criticality, or neutron multiplication factor, $k_{e f f}$ of a reactor is defined by the sum of all the reaction rates of processes which produce neutrons (in this case fission), divided by the rate at which neutrons are destroyed via absorptions, leaks, etc.

$$
k_{e f f}=\frac{\Sigma v_{i} n_{i} \sigma_{f} \phi}{\Sigma v_{i} n_{i} \sigma_{c} \phi+\Sigma n_{i}\left(\sigma_{c}+\sigma_{f}\right) \phi}
$$

where $\phi$ is the neutron flux, $v$ is the average neutron multiplicity, $\sigma_{f}$ and $\sigma_{c}$ are the fission and capture cross sections of the $i$ th isotope present in the core. The importance of a given nucleus is therefore proportional to the number of atoms in the core, $n_{i}$, which at the beginning of the cycle consists of just the initial fertile and fissile isotopes in the fuel. At the end of the cycle however, after a range of new nuclei have been synthesized. Many neighbouring nuclei to the fissile and fertile isotopes will have accumulated, along with the many fission products. Ideally, we would like to know the sensitivity of the criticality to the cross sections of each isotope present.

\subsection{Reaction rates in the core}

In general, reactor physics neutronics simulations aim to extract detailed information on the neutron flux and, hence reaction rates, in different spatial regions of the core. Over long time scales, these reaction rates can then be used to calculate the reactor fuel composition as a function of time to find out the quantities of new isotopes synthesized. There is a complicated network of reactions and decays which link the creation and destruction of several hundred nuclei during the irradiation of the fuel in the reactor. The goal is to understand the isotopic composition of the spent fuel with a view to either recycling the useful fissile and fertile material, or of disposing of it in, interim or long-term geological storage.

Over shorter time-scales, the reaction rates are essential for determining core power distributions. From these power distributions fuel and coolant temperatures can then be calculated using thermalhydraulic models for the heat transfer between fuel and coolant. Under nominal power conditions, the reactor must not pass any of the margins which could impact on its safe running. Reactor cores are designed to be auto-stable where any unexpected increase in the power will lead to an increase in temperature and immediate decrease in the power (i.e. $d k / d T$ is strongly negative).

Furthermore, determining how the reactor power will behave in transient situations such as startup, shutdown, and accidental scenarios is crucial, requiring kinetic calculations that allow the reactor power and temperatures to evolve as a function of time. Again, the accuracy of the cross section data have a direct impact on the accuracy of the simulations.

Since the performance of a future reactor will be diminished if the safety margins must take into account the inaccuracy of the nuclear data, then better measurements will allow these safety margins to be reduced. Hence, it is possible to directly increase the performance of the reactor at very little extra cost by performing nuclear data experiments, since the cost of these experiments is minuscule when compared the cost of a prototype future reactor.

\section{The limitations of direct measurements}

In the actinide region about half the nuclei (see figure 1) have target activities greater than $10^{9} \mathrm{~Bq} / \mathrm{mg}$. This makes direct measurements particularly problematic due to radioprotection concerns. It is very difficult to obtain the material for the target, difficult to safely manufacture the target and difficult to place it in an experimental setup.

To overcome these problems, indirect methods of measuring reaction cross sections have been developed[2,3], which will be discussed later.

\subsection{Nuclei at equilibrium}

Most of the actinides for which it is difficult to measure cross sections directly are nuclei which achieve an equilibrium during reactor irradiation, since they have half lives 


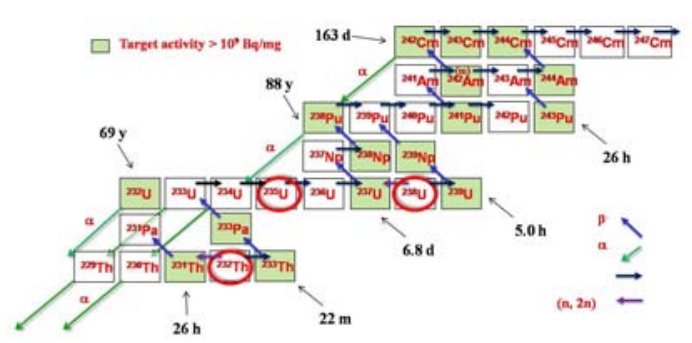

Fig. 1. Important nuclei in the actinide region. Nuclei with target activities greater than $10^{9} \mathrm{~Bq} / \mathrm{mg}$ are shaded green.

which are shorter than the total irradiation time (3-5 years). They are continually produced by nuclear reactions in the core and continually destroyed via radioactive decay (or other reactions). If the lifetime of a nucleus produced via a neutron induced reaction in the core is significantly shorter than the irradiation time of the fuel, then the nucleus will reach an equilibrium concentration since its rate of destruction via decay will be equal to its rate of production via neutron induced reaction. For a short-lived nucleus, $b$, produced by a reaction on its parent, $a$, it's rate of production at equilibrium will equal its rate of destruction:

$$
n_{a} \sigma \phi=\lambda n_{b}
$$

We note that the equilibrium concentration of nucleus, $b$, in the core will therefore inversely proportional to its decay constant, $\lambda$, and thus proportional to its half-life, the shorter the half-life the lower its equilibrium concentration will be. This implies that measuring cross sections for nuclei with very short lifetimes is less important. As a general rule, this is true, however, there are many notable exceptions, where nuclei that exist in very small concentrations have a massive impact from a reactor physics point of view.

\subsection{Important short lived nuclei}

The most well known example of such a nucleus is the fission product ${ }^{135} \mathrm{Xe}$, which has a half life of only 9 hours. In a typical PWR of 90 tons, the equilibrium mass of ${ }^{135} \mathrm{Xe}$ will be only around 25 grams. However, the neutron capture cross section for ${ }^{135} \mathrm{Xe}$ at thermal energies is enormous, over $10^{6}$ barns, due to the presence of a single resonance which lies almost exactly at the neutron binding energy. Decay of this nucleus (a strong neutron poison who's presence reduces reactivity) may cause problems at reactor shutdown.

Another example is the nucleus ${ }^{232} \mathrm{U}$ which is only produced in tiny quantities in a reactor with thoriated fuel via the $(n, 2 n)$ reaction. It has a half life of 69 years, so never reaches equilibrium. However, the radioprotection consequences of the presence of even trace amounts of ${ }^{232} \mathrm{U}$ in the spent fuel are highly significant. Since the decay chain of this nucleus ends rapidly at the $2.6 \mathrm{MeV}, 2^{+}$to $0^{+}$gamma transition in ${ }^{208} \mathrm{~Pb}$ the spent fuel must be handled remotely. (The thickness of the shielding required to attenuate significantly a flux of $2.6 \mathrm{MeV}$ gamma rays is considerable.)

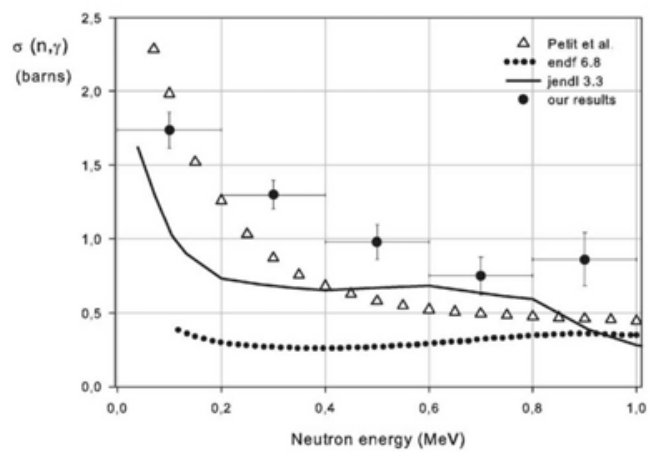

Fig. 2. Indirect measurement of the neutron induced capture cross section on ${ }^{233} \mathrm{~Pa}$ using the surrogate method.

\subsection{Limitations of the nuclear data}

As previously mentioned, for certain nuclei the cross section data are sparse (limited energy range), can have large uncertainties $(>20 \%)$. For certain nuclei in the actinide region cross sections can be measured indirectly using the surrogate method[2]. This indirect method relies on producing the compound nucleus using transfer reactions involving charged particles, the energies of which are measured experimentally, thus the compound nuclear excitation energy is known. Partial decay probabilities can then be determined for the various exit channels. However, there are limits to the technique, particularly for indirect neutron capture measurements since there are no easy ways to discriminate experimentally between gamma rays from excited fission fragments and gamma rays from radiative capture.

This method was used with success to measure the fission and capture cross sections of the most important equilibrium nucleus involved in the thorium cycle, ${ }^{233} \mathrm{~Pa}[4,5]$. The results from our measurement of the capture cross section between 0 and $1 \mathrm{MeV}$ using the surrogate method are shown in figure 2. It can be seen two evaluated data bases ENDF6.6 and JENDL3.3 differ by approximately a factor of two. The principle reason for this is that the data bases rely on calculations of the cross section using statistical methods such as Hauser-Feshbach theory, but with the level densities of the residual nucleus calculated by different semi-empirical models.

\section{Level densities and improvements of reaction cross section calculations}

\subsection{The Hauser-Feshbach statistical formalism}

In Hauser-Feshbach theory, determination of the cross section for a given compound nuclear reaction relies on knowledge of the compound nuclear formation cross section, the transmission coefficients of the ingoing and outgoing particles, and the level densities in the residual nuclei[6,7]. The compound nuclear formation cross sections and transmission coefficients are known fairly accurately from optical 


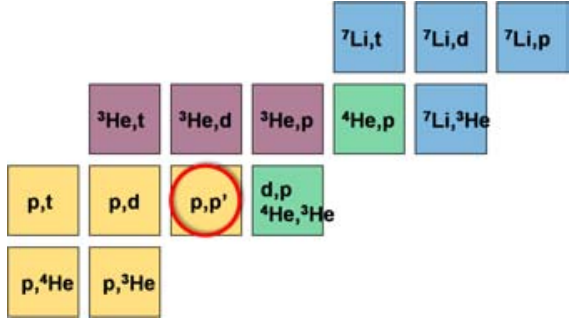

Fig. 3. Residual nuclei on the Segre chart which can be reached via transfer reactions involving only charged particles in the exit channel. The target nucleus is marked with the red circle.

model calculations and nuclear data obtained near the valley of stability. However, the major part of the difference between calculations arises from the lack of knowledge of level densities[8]. Level densities as a function of excitation energy are not well known in the actinide region and the use of semi-empirical formulas to predict level densities for across the nuclear chart, such as the back shifted Fermi gas model or constant temperature model often over or underestimate level densities by a factor of two compared two experimental values[9]. For this reason, theoretical calculations of cross sections do not reproduce measured cross sections very accurately.

In addition to the problems of theoretical approaches to cross sections in the actinide region, there are also problems for cross sections of reactor structural materials. While most neutron capture cross sections are measured with a high degree of accuracy, there exists a problem for the minor exit channels such as $(n, p)$ and $(n, \alpha)$ reactions. Even though these reactions have very tiny cross sections, the production of hydrogen and helium gas in reactor structural materials over time can change their chemical composition and hence affect the material properties and ultimately structural integrity. Again, improved knowledge of level densities of residual nuclei in these reactions would be very helpful information.

\subsection{Program of Level Density Measurements}

At low excitation energies, discrete line gamma-ray spectroscopy can provide information on the excited nuclear states and hence give information on level densities. However, as excitation energies become higher it becomes quickly impossible to resolve discreet states. The level density can also be obtained at the neutron binding energy if neutron resonance spacings are known. However, in the region in between level densities must be measured in a different manner by using the Oslo method[10]. The level densities can be extracted as a function of excitation energy using this technique based on the Brink-Axel[11] hypothesis and involves measuring particle- $\gamma-\gamma$ correlation spectra.

We therefore intend to start a program of measurements of level densities in the actinide region at the Oslo cyclotron as a collaboration between the university of Oslo, IPN Orsay and CEA Saclay. The Cactus array provides an ideal instrument to perform such studies due to its very high gamma-ray photopeak detection efficiency of $\approx 15 \%$ and the ability to detect the outgoing charged particles induced via transfer reactions in silicon $E-\Delta E$ telescopes. The goal is to firstly test the validity of Hauser-Feshbach cross section calculations to see if they can reproduce cross sections that have already been measured to a high degree of accuracy (e.g. $\left.{ }^{232} \mathrm{Th}(n, \gamma)[12]\right)$ by providing them with measured level densities for the residual nuclei. Eventually, a picture of the systematic level density variations in the actinide region could be built up, allowing improvement of theoretical calculations for nuclei where nuclear data are sparse or non-existent. Figure 3 shows the residual nuclei that it is possible to produce from a transfer reaction involving only charged particles. If ${ }^{7} \mathrm{Li}$ beams can be used (although they may bring in too much angular momentum into the compound system), then it will be possible to reach around $90 \%$ of the actinides shown in figure 1 with only six targets which are reasonably easy to fabricate and obtain $\left({ }^{232} \mathrm{Th},{ }^{238,235,233} \mathrm{U},{ }^{237} \mathrm{~Np}\right.$ and $\left.{ }^{239} \mathrm{Pu}\right)$.

Moreover, in certain instances the Cactus array may allow simultaneous measurement of capture cross sections via the surrogate method, provided that: (i) compound nuclear excitation energies are below the fission threshold of the equivalent neutron-induced reaction to prevent contamination from fission fragment gamma rays, and (ii) only charged particle channels are open, since NaI detectors cannot discriminate perfectly between neutrons and gamma rays and have a large neutron absorption cross section.

\section{References}

1. The Generation IV International Forum: http://www.gen-4.org/

2. J.D. Cramer, H.C. Brit, Nucl. Sci. Eng. 41 (1970) 77

3. J.N. Wilson, B. Haas, et. al, Nucl. Instrum. and Meth. A511 3 (2003) 388-399

4. M. Petit, et. al, Nucl. Phys. A735 (2004) 345

5. S. Boyer, D. Dassie, J.N. Wilson et. al, Nucl. Phys. A775 (2006) 175-187

6. W. Hauser and H. Feshbach, Phys. Rev. 87 (1952) 366

7. A. Wallner, B. Strohmaier, and H. Vonach, Phys. Rev. C51 (1995) 614

8. A.V. Voinov et. al, Phys. Rev. C76, 044602 (2007)

9. Till von Egidy and Dorel Bucurescu, Phys. Rev. C72 044311 (2005)

10. M. Guttormsen, T. Ramsoy, J. Rekstad. Nucl. Instrum. Meth. A225 (1987) 518

11. P. Axel, Phys. Rev 126 (1962) 671

12. G. Aerts, et. al, Phys. Rev. C73 054610 (2006) 